\section{Simplification and Variation in TEM Focus Techniques}

\author{
Steve Chapman \\ Protrain, Buckingham, England \\ protrain@emcourses.com
}

In order to understand the focusing action of a TEM objective lens, a simple imaging system (figure 1) is best considered. This system consists of an objective lens and a single projector. In operation, the projector is adjusted as required within the total imaging system to achieve a magnification, M2, on the screen. This results in a focal length of $\mathrm{F} 1$, with the lens seeking to find an image in the position M1. If the objective lens does not place the image at M1 the result on the screen is an out of focus condition. The objective lens may have produced an image short of M1, overfocus, or beyond M1, underfocus. Therefore it may be said that the action of focusing an image is accomplished by an adjustment to the objective focal

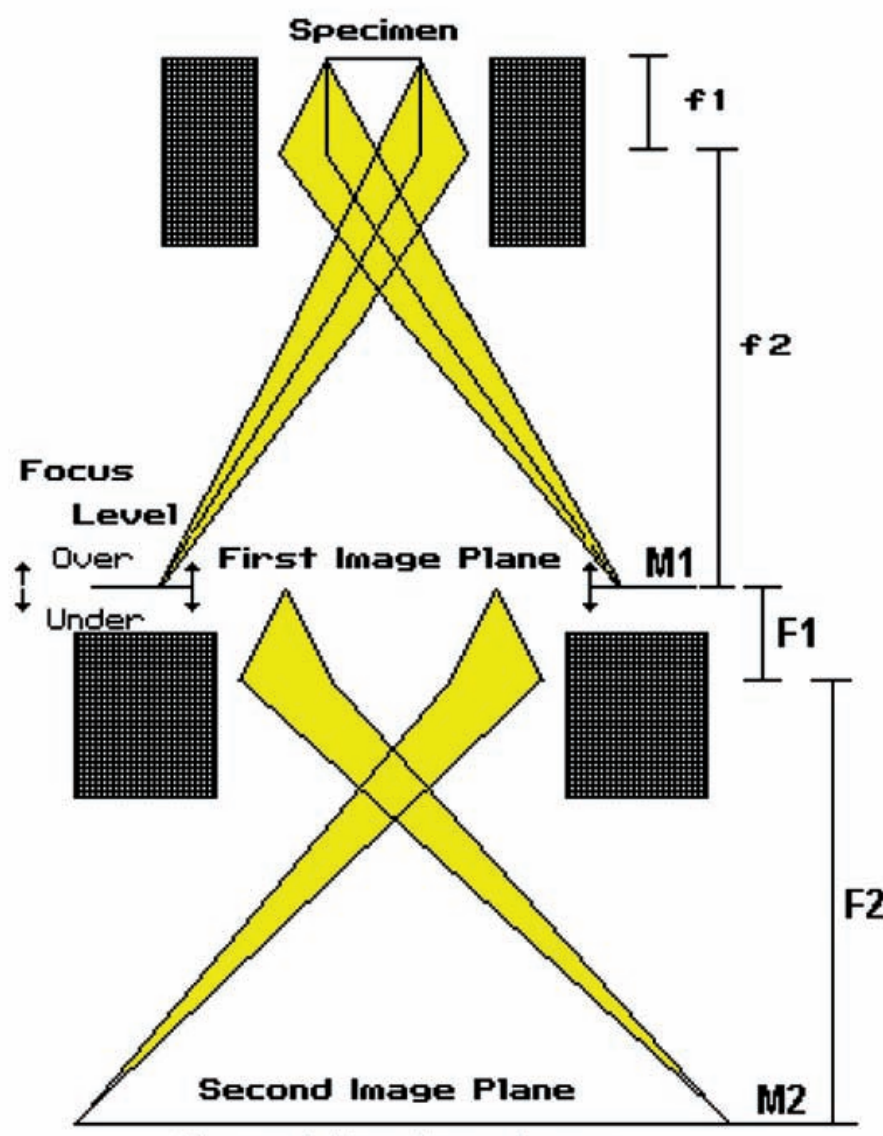

Second Specimen Image

Figure 1

length to coordinate the first image plane with the focal length of the lens following - usually know as the diffraction lens in a multi lens imaging system. From the diagram, focus is achieved when the objective lens places the image at M1. Once the lenses have been adjusted to attain the desired magnification and the focal lengths are co-ordinated, the image remains in focus regardless of the number of lenses and the distance to the screen or recording media. This condition of retaining focus over a considerable distance is known as Depth of Focus and it is considered to be infinite in a TEM. Depth of Field, is related to the thickness of the specimen in focus at any one time, about 2 microns in a TEM.
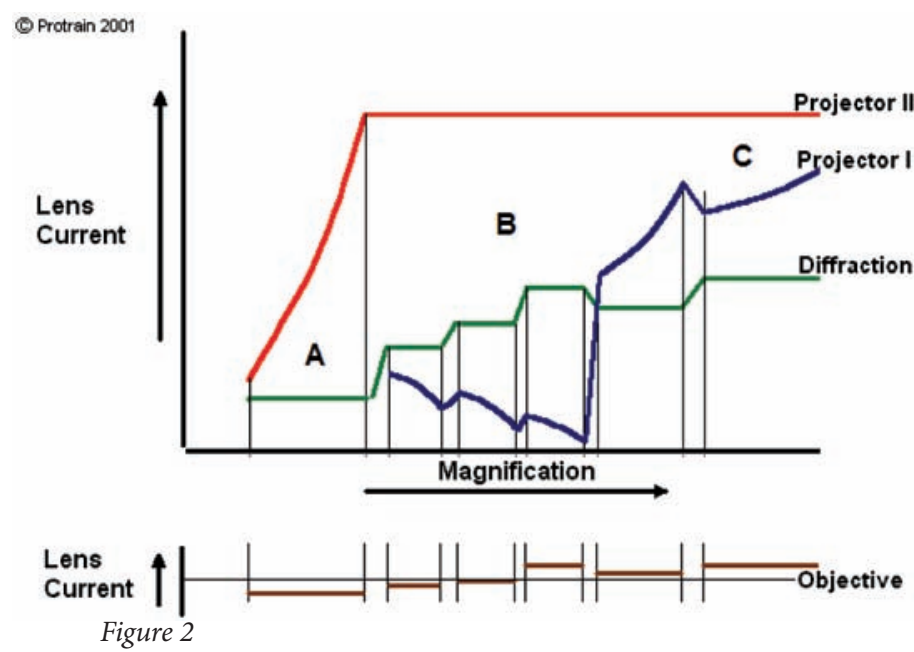

From the above we see that a microscope only requires a focus correction when the diffraction lens current is changed. In modern instruments, an objective lens current change may only be demanded as little as four times over the complete magnification range.

Observation of the diffraction lens current as the magnification is adjusted will indicate the points in the range where a focus correction is required. Thus a more accurate focus and astigmatism correction may be made by moving to the top end of each range where the diffraction lens current is static and then reducing the magnification to lower levels within this range prior to recording an image. A simple four-lens imaging system is plotted in figure 2, which demonstrates that a constant diffraction lens current requires a constant objective lens current.

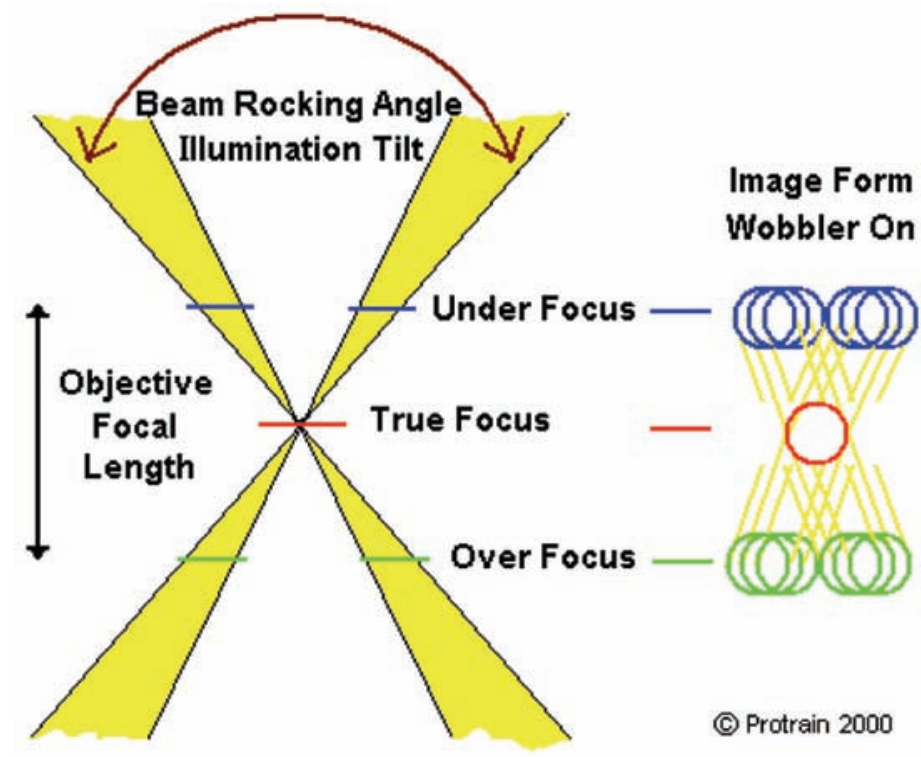

Figure 3

As an aid to finding true focus, most instruments are fitted with a wobbler focus aid, figure 3 . This feature takes advantage of the fact that if the electron beam is rocked over a small angle about a point on the specimen surface, when the surface is out of focus, the image moves or splits into two. It should be noted that although this system assists in the determination of true focus, this condition may not be ideal for the optimum presentation of the specimen. For this reason wobbler focus is best used as a constant set point before offsetting the focal conditions by a pre-determined amount 


\section{Value and Excellence in SEMs}

VEGA Scanning Electron Microscopes

Fully automated PC-controlled SEMs

designed for a multi-user environment.

High performance 4-lens optics provides high resolution, high depth of focus, extremely large field of view as well as optimum working conditions for any application.

High working vacuum and variable pressure operation up to $2000 \mathrm{~Pa}$.

Original patented Low Vacuum Secondary Detector for true secondary electron imaging under low vacuum.

Full range of precision-engineered specimen chambers and stages.

Sophisticated yet easy-to-use software for microscope control and image processing \&t evaluation.

Network operations with built-in remote control and diagnostics.

3D surface reconstruction using $3 \mathrm{D}$ beam technology.
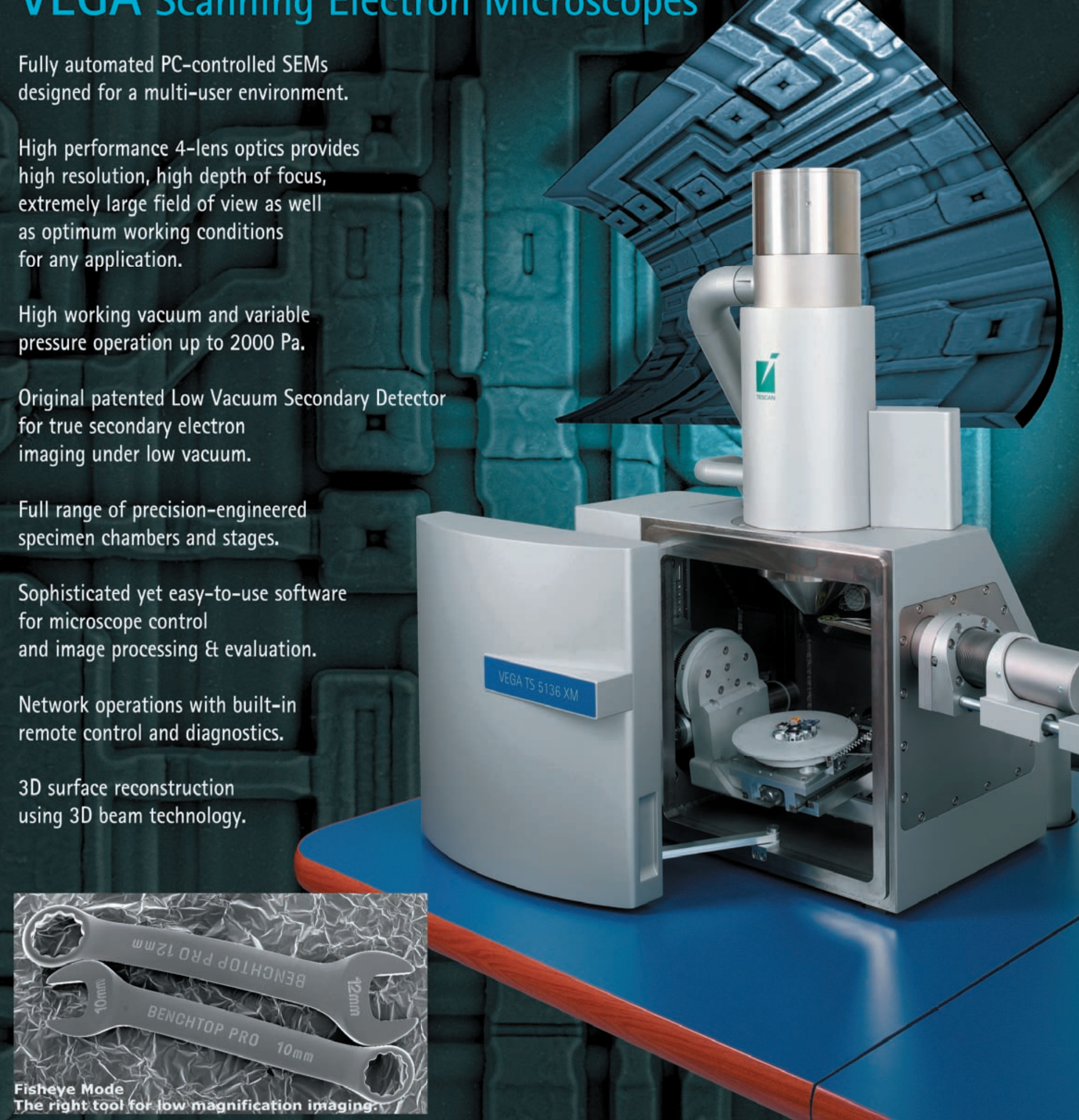


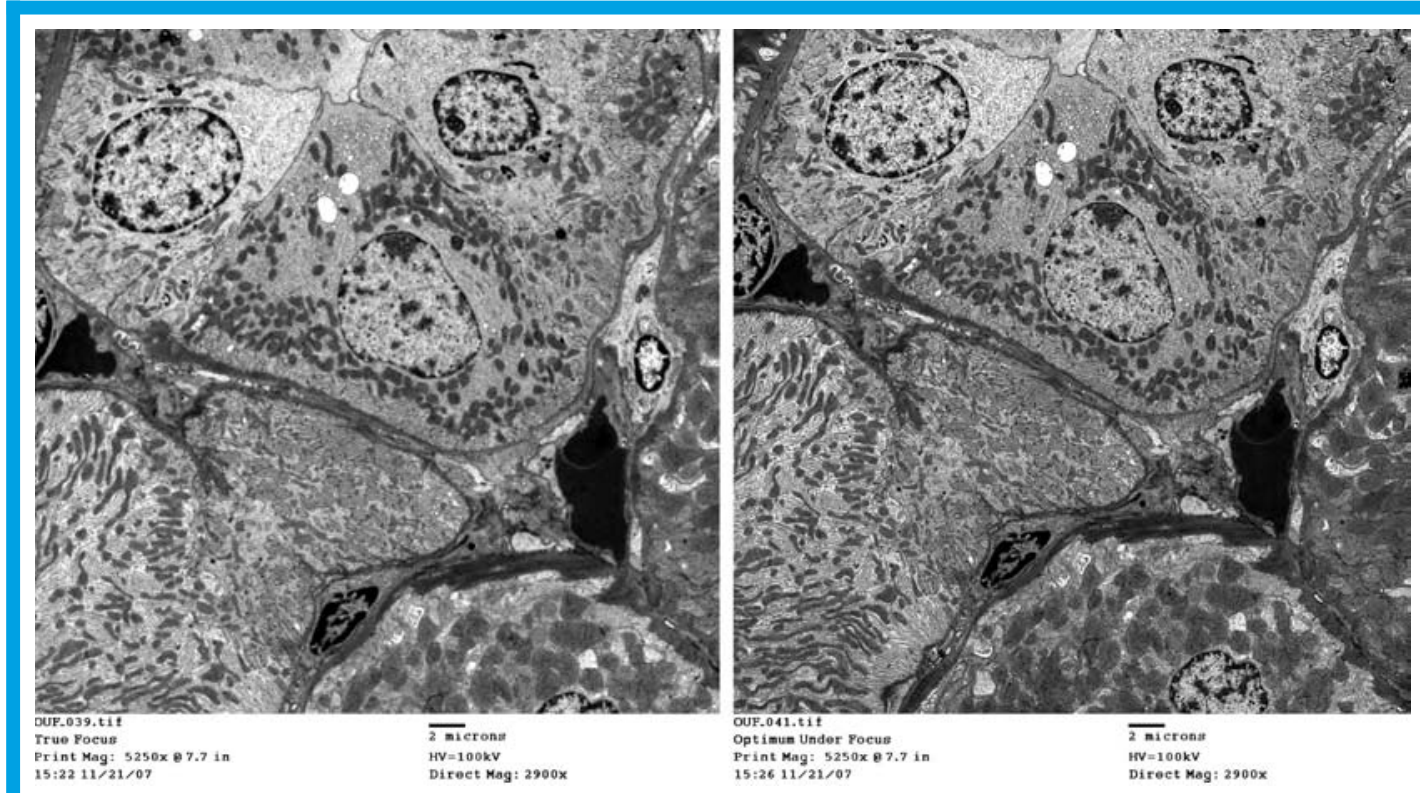

Fugure 4

to attain optimum image recording conditions.

The high performance lens used to focus the image in a transmission electron microscope, the objective lens, is usually designed to achieve a resolution level better than $0.2 \mathrm{~nm}$. For a lens to achieve this capability, its inherent contrast attributes vary in relation to the focus level that is set. At true focus, the lens maximises contrast at the sub nanometre level, creating images with poor contrast when the structures are tens of nanometres in size. In order to achieve higher contrast with these larger structures the objective lens contrast mechanism is optimised by moving underfocus from true focus.

The ideal under focus setting, with regard to maximizing appreciation by the human eye, is termed optimum under focus, or o.u.f. In this case, image visualation is enhanced by adjusting the objective lens to under focus the dominant structures within the sample. Some instruments have the facility to automatically set a degree of under focus when the wobbler key is released but this is a generic programmed step that may be considerably improved upon in most applications.

In material science, the degree of under focus from wobbler focus is minimal, compared to that required for biological material, where specimen scattering leading to image contrast may be extremely variable. With plant material, the o.u.f. may be a considerable distance from wobbler focus with widely spaced organelles, whereas with dense organelles as found in kidney, the o.u.f. changes

\section{Optimum Under Focus}

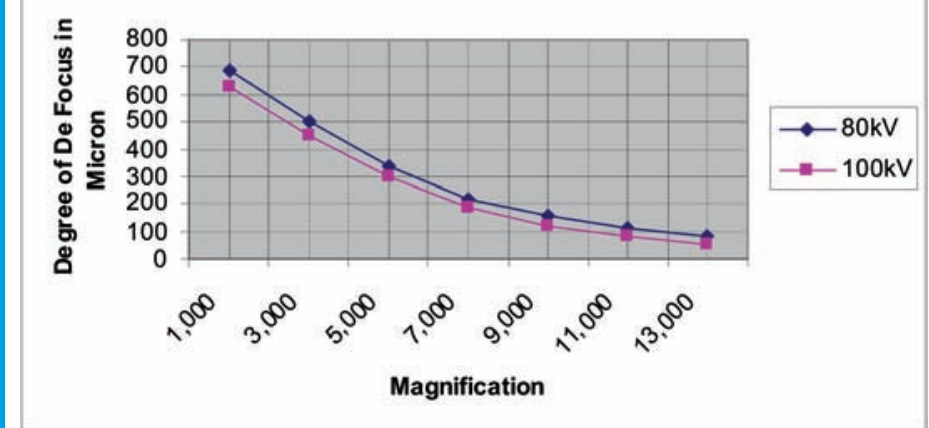

Figure 5 be followed.

Set wobbler focus and, either by counting focal steps, or reading off the focal values provided by the manufacturer, the focal change is recorded as the focus current is reduced to the point where the operator judges the image contrast to be at its best. To confirm the setting, micrographs are recorded either side of this point at suitable focal steps, three slightly nearer to focus, one at the operators selected point and another a little further underfocus. The micrographs are printed in the normal fashion, the most desired image selected, and its underfocus value noted. This procedure is repeated over the range of magnifications used with the particular material. Finally o.u.f. vs. magnification is plotted, as in figure 5, for that particular material and set of conditions.

While there is a degree of assistance with determining focus, or o.u.f., when using the focus wobbler, there is not usually a procedure on commercial instruments that assists with the determination of objective lens astigmatism. The operator concentrating on a high contrast area within the specimen may accomplish both optimum focus and correct astigmatism compensation. It is important that the area is not directional as this confuses the task. A focus adjustment that provides maximum contrast will be at or near to o.u.f. and from that point the stigmators should be adjusted in turn to the level where they too enhance the contrast in the area of interest. Operators should be aware that any change made to objective lens focus will most likely require a concomitant change of objective lens astigmatism compensation.

Naturally when discussing focus and image quality, image contrast must also be addressed. In a transmission electron microscope, image contrast is usually a combination of amplitude and phase contrast or diffraction contrast. In the investigation of biological thin sections, amplitude contrast is the main consideration.

Recognizing that an increase in accelerating voltage has a considerable effect upon image contrast, biological transmission electron microscopy was initially carried out at 50 or $60 \mathrm{kV}$, depending upon the $\mathrm{kV}$ range of the instrument. However in order to introduce the minimum amount of damage to the specimen, and to obtain suitable illumination levels for operation, it is advantageous to use the highest accelerating voltage that still enables focus to be attained 
comfortably. Visual contrast is of no importance, other than to view and focus the specimen: the screen is a means to end not the result! Any contrast loss on the screen compared with low $\mathrm{kV}$ techniques, will be more than corrected on the print using optimized image collection procedures, be it digital or photographic. In general, one $\mathrm{kV}$ step higher than conventionally used in a laboratory is not excessive. Improvements in embedding media and a greater awareness of the improvement in image quality through the use of higher accelerating voltages, have resulted in a move towards greater use of these higher voltage levels. Whilst most biological thin section microscopy may be carried out with adequate contrast when operating at $80 \mathrm{kV}$, it is not uncommon for current work on thin sections to be carried out at 100 or $120 \mathrm{kV}$. Operators are recognizing that artifacts may be introduced into an image through too low an accelerating voltage, and the true interpretation of a stained area may become confused because there is too much contrast.

In material science, particularly when working with metals, the tilting system plays a large part by adjusting the orientation of the specimen to maximize the appropriate diffraction contrast. It is therefore essential to ensure that the stage is correctly set at the eucentric position before recording an image and that before tilting the tilt speed is at the desired level.

As mentioned earlier, conventional design criteria for an objective lens are directed toward the highest resolution. High resolution dictates the shortest focal length and minimum spherical aberration. However for the biological scientist the ideal requirement is a longer focal length, where the resulting increase in spherical aberration aids image contrast-a point recognized by some manufacturers who produce a biological version of their instruments.

With eucentric side entry systems under normal operating conditions it is very important to set the eucentric point as that sets the focal length and magnification of the objective lens, on which following lenses build. Failure to set this condition will result in an inaccurate magnification range. However with a eucentric system the variation in focal length that the eucentric height, or $Z$ prime, adjustment offers enables a useful change in focal length either, in one direction, to increase the ultimate resolution, or in the other direction, to increase the image contrast.

Adjusting $Z$ prime to raise the specimen yields a longer focal length with the result that the magnification range is lowered, but, interestingly, the contrast level is increased, even when using the same objective aperture. Figure 6 shows how a longer focal length effectively reduces the angular aperture of the objective, increasing contrast. The specimen is moving up, out of the lens, when the focus control must be turned anticlockwise to

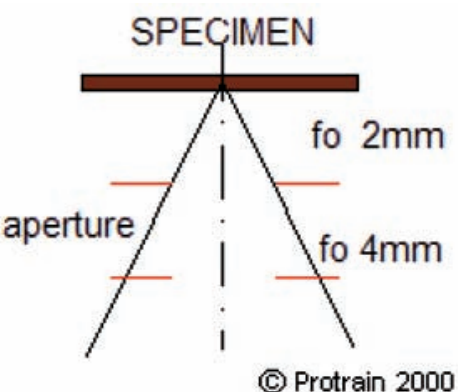

Figure 6 reach focus. Conversely, lowering the specimen further into the lens field will facilitate those striving to push the instrument to its limit to attain higher resolution images, as aberrations decrease with higher lens strength. With the objective lens working at a higher current in order to focus the lowered specimen, there will be an increase in magnification. In both of the above cases recalibration of the instrument's magnification will be required-a small chore

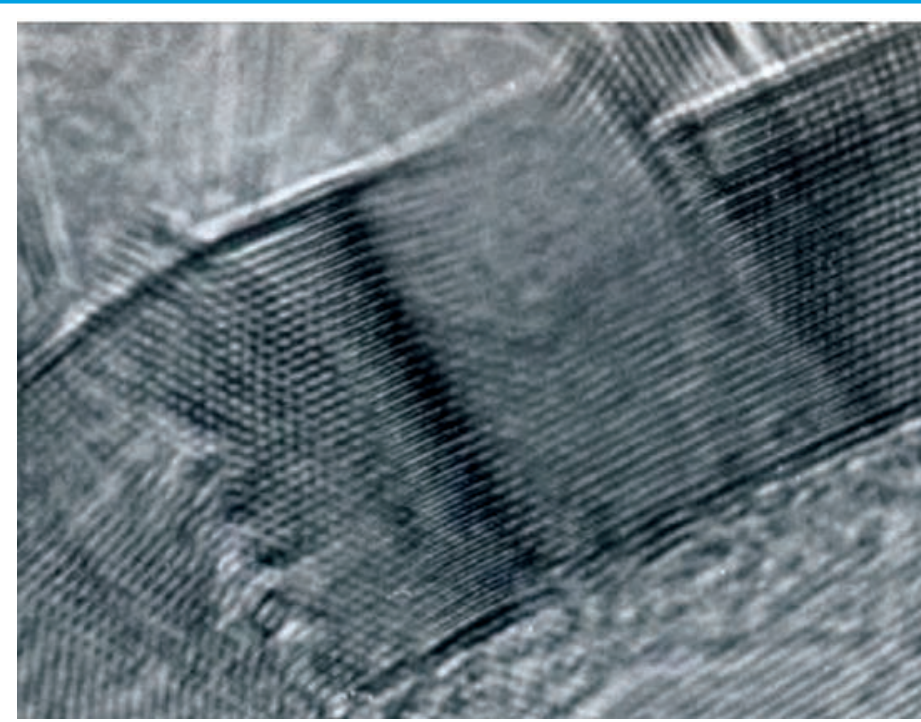

Figure 7

when the results attained offer better contrast in one stage $\mathrm{Z}$ direction and considerably higher resolution in the other.

The Micrograph in figure 7 was taken on an instrument guaranteed for $0.344 \mathrm{~nm}$ resolution. Using $Z$ prime to adjust the eucentric stage to its lowest position, we see the half lattice of $0.172 \mathrm{~nm}$ resolved in the central area of the image.

In summary, transmission electron microscopes have a number of variable features and if optimised for the task in hand they may often allow the operator to attain a quality of result that is not normally possible.

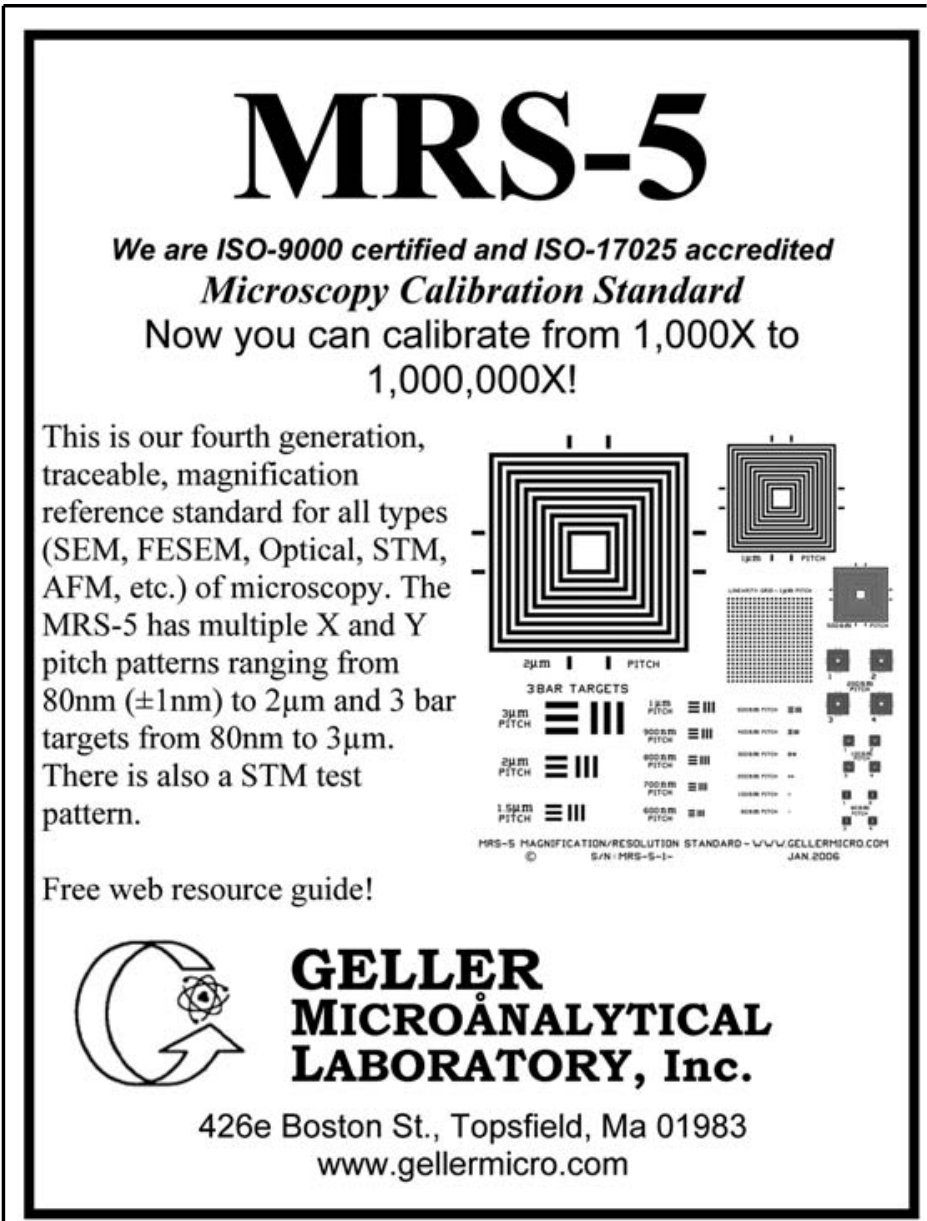

